present understands why there has been such a rapid decrease of dust production in the three days between the two Vega encounters.

Dust appears to have played havoc with several of the Vega experiments. A lowfrequency plasma detector, based on an antenna stretched between one of the booms projecting from the spacecraft and the solar panels, went out of action just before the first encounter, to the resigned annoyance of the group responsible. On the less successful encounter of Vega 2, several instruments went out of action before the encounter. One speculation is that the spacecraft may have become electrically charged in such a way as to affect the operation of instruments requiring a stable reference voltage.

The encounter of Vega 2 was also complicated by a pointing error, which for most of the approach had the cameras locked on to a bright patch of scattered sunlight offset from the nucleus of the comet. The most serious consequence was that the automatic microprocessor-based program relating the exposure of each frame to the brightness of the aiming point consistently over-exposed the nucleus. In a nail-biting emergency, the error was corrected only ten minutes before the encounter with the help of a back-up guidance system.

The general opinion of experimenters and managers is that the project has been outstandingly successful. Those gathered at the Institute of Space Science of the Soviet Academy of Science, which has mounted the exercise, have been quick to pay tribute to the international collaboration on which the project has been based.

Collaborators come from France and West Germany in Western Europe and from Czechoslovakia, Hungary and Yugoslavia in Eastern Europe. Although the United States is not formally involved in a project mounted when relations between the Soviet Union and the United States were in the doldrums, Professor J.A. Simpson from the University of Chicago has flown a novel dust detector on the identical Vega spacecraft at the invitation of Professor R.Z. Sagdeev, the director of the Soviet project and the institute at which it is based.

By common consent, the Deep Space Tracking Network of the US National Aeronautics and Space Administration (NASA) had also provided invaluable assistance both during the preliminary encounters of the Vega spacecraft with the gravitational field of Venus at the end of last year and in refining the trajectory of the vehicles.

Sagdeev recalls the Jet Propulsion Laboratory in California calling up one night with the question why one of the two Vegas had apparently vanished from the radio sky.

Halley's comet

\title{
Telling the truth in real time
}

\section{Moscow}

As this monumental city last Wednesday watched its four-month covering of snow turn to grey slush, the builders of the first of four probes to Halley's comet were celebrating (mostly in the abstemious Gorbachev style) their successful first look at that eroding dirty snowball in the sky. But the more striking achievements may be the informality of the international collaboration on which the Vega project was based and the frankness with which all the details of the project were displayed.

To say that the outcome confirms the dirty snowball picture of a comet, that of a lump of frozen water with embedded dust, true though it might be, would be to diminish the occasion. Getting within $8,000 \mathrm{~km}$ of an object nine light-minutes away may be child's play these days, while Voyager has shown that instruments can be made to function at least a decade after being built, but who can suppress childish pleasure that five years of planning for an hour or so of proximity to Halley should, in the end, succeed?

There were, in fact, two Vegas and thus two close encounters, on Wednesday last week and on Sunday. As launched (in December 1984), the two spacecraft were identical. Each of them dropped a balloon into the atmosphere of Venus before being deflected towards the retrograde orbit of Halley, still last week between the orbits of the Earth and Venus (but with the Earth on the other side of the Sun). At the Soviet Academy of Science's Institute of Space Sciences, the interval was spanned by a kind of perpetual polyglot symposium interrupted only by visits to other laboratories and the need for lunch.

Strictly, there have been two on-going

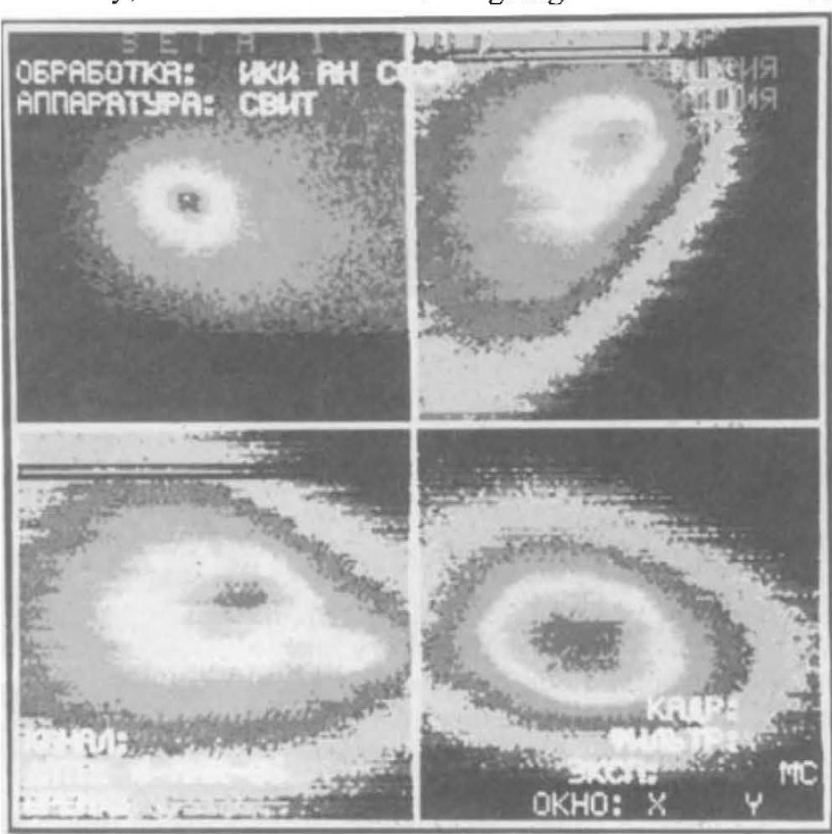

symposia. The experimentalists have been crammed with their microprocessors into a ground-floor room about $30 \mathrm{~m}^{2}$ dominated by a full-scale model of Vega. Each experiment has been able to monitor its own data stream in real time; most, almost as a matter of pride, have thought it seemly to produce an instant analysis and interpretation as well. The Hungarian image processers were back-to-back with the Austrian recorders of the magnetic field. The Czechoslovak and US dust particle experiments were within whispering distance of each other (with the West Germans across the corridor). The French team taking ultraviolet spectra have been almost inaccessible in their corner.

The other symposium, on the floor above, has been for dignitaries, and has been recognizable by the bottles of mineral water on the tables. (Local opinion holds that "something is going to happen" when the bottles are changed; "they know we'd drink them if they left them there too long".) The dignitaries have included Dr Fred Whipple, lately retired from the Harvard-Smithsonian Astrophysical Observatory, the originator of the dirty snowball picture, Dr Thomas Donahue, chairman of the US National Academy's Space Sciences Board, delegations from the European Space Agency (ESA), the US National Aeronautics and Space Administration (NASA) and from the Space Sciences Institute at the University of Tokyo and vice-presidents of the Soviet Academy, who dropped in from time to time.

During both encounters with Halley's comet, this symposium has been kept thoroughly up to date by a display on two substantial video screens of data as re-
False-colour images of Halley's comet (shown here in black and white) from Vega 1 at around the time of closest approach at 07.20 GMT on 6 March, with four different filters. Although the top left-hand image shows a small peak of intensity (which appears white) near the centre, this cannot be identified with the nucleus of the comet, but is rather an artefact of the colour-coding system. The double structure of the image immediately below, in the near infrared, may signify the jet of dust encountered by Vega 1 . The distance across each frame is about $1,500 \mathrm{~km}$. 
ceived and analysed by the experimenters in virtually real time. Professor Albert Galeev provided a running commentary, doing his best to interpret data as they appeared and succeeding reasonably well except when the projectionists switched displays in the middle of a sentence. (A Hungarian image processor says he had not been asked to provide this frill until a month ago.) It is an interesting and moving experience to see mass spectrograms of the products of the impact of single cometary dust particles on a silver target literally before the West Germans who built this ingenious apparatus had a chance to censor them.

Formally, the six-day symposium was a meeting of the inter-agency coordinating group bringing together the US and European space agencies, an amalgam of the two agencies in Japan and that from the Soviet Union. Academician R.Z. Sagdeev, the driving force behind Vega and the manner of its public presentation, wanted to use the occasion for hammering out a further programme of collaboration, offering to form a working group to say what should be done next before everybody took flight for Darmstadt and the Giotto encounter. But neither NASA nor ESA was ready for this proposal, suggesting instead a formal discussion at a meeting planned for Padua in November.

Apparently there is a plan to move from Padua to Rome for a public presentation of the Halley data, including an audience with the Pope, to whom each agency is expected to provide its four best pictures of the comet, possibly on 7 November. Those making the arrangements appear to have overlooked the significance of the date, the anniversary of the October $\mathrm{Re}$ volution. Sagdeev wondered whether the Pope might be persuaded to celebrate mass for the occasion.

For the rest, the dignitaries behaved as dignitaries do on these occasions. Their congratulations have been full-throated, not merely for the success of the two Vegas but for the openness of the proceedings of which they have been a part. The institute's staff has been surprised to find the proceedings televised live from the "data analysis room", which is said to be unprecedented. But what about the Party Congress? "That's the other exception."

The good-humoured meeting has also been sadly depressed about the shuttle tragedy, wondering what the effect will be on the US space programme. NASA officials say that it has been decided to plan on the assumption that the shuttle will be operating again after a delay of twelve months, and that priority will then be given to launching the Galileo mission to Jupiter and the Hubble space telescope. The snag, it seems, is that "it's costing us $\$ 10$ million a month to keep things on hold".

John Maddox

Halley's comet

\section{Japan's probe rocked by dust}

\section{Tokyo}

"IT's great" was how researchers at Japan's Institute of Space and Astronautical Science (ISAS) reacted to news that their space probe Suisei (comet) had safely passed its point of closest approach to Halley's comet. Suisei was twice shaken by collision with high-velocity dust particles as it came within $150,000 \mathrm{~km}$ of the some rapid calculations as to just how big the particles must have been to rock the $135-\mathrm{kg}$ probe, bearing in mind that their relative velocity is something like $73 \mathrm{~km}$ per second. Initial estimates were that the dust weighed about $10 \mu \mathrm{g}$; but recalculation came up with a figure of $1-2 \mathrm{mg}$, giving the particles diameters of $1-2 \mathrm{~mm}$. If this proves correct it is a very big sur-

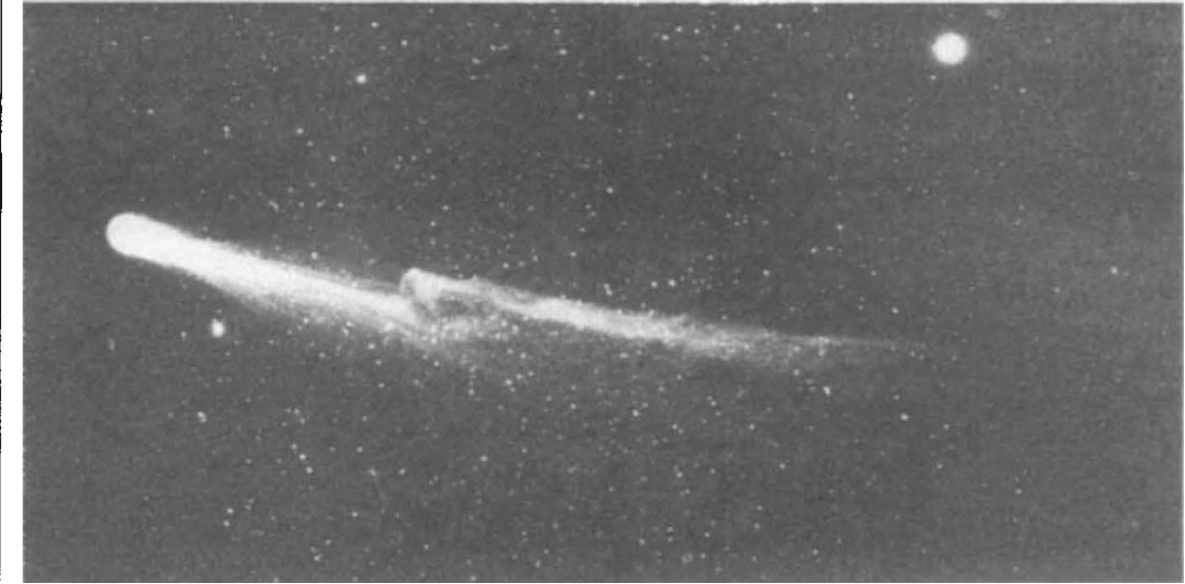

Sun side of Halley at 22.06 on Saturday, 8 March (Japan time) but in neither case was it thrown sufficiently out of orientation to affect data collection.

News of the collisions and data from the encounter did not reach Japan until Sunday, for the probe was on the wrong side of the Earth to communicate with ISAS's new deep-space observation centre at Usuda in Nagano Prefecture, west of Tokyo. Last commands sent to Suisei on Saturday were for the probe to continue to make observations with its ultraviolet camera and its ion-energy analyser according to programme. From Saturday evening until Sunday morning, signals were picked up at the US National Aeronautics and Space Administration (NASA)'s space observation centres in Spain and California, suggesting that all was going well. Then when Suisei came back round to the Japan side of the Earth, all data collected during the encounter were taken out of memory and transmitted to Japan.

The first and biggest surprise was that the probe had been shaken by collisions with dust particles just before the point of closest approach. The first collision at 20.54 knocked the rotation axis of the probe relative to the Sun out by $0.48^{\circ}$; the second at 21.26 added another $0.24^{\circ} \mathrm{de}$ viation. But neither was sufficient to affect observations of the comet. The first impact was also powerful enough to slow down the satellite's spin period of 9.184 seconds by 0.027 seconds; the second, presumably to a different part of the structure, had a scarcely noticeable effect.

News of the dust collision triggered prise and may be bad news for the European spacecraft, Giotto, which is going very much closer to Halley later this week. "We don't know whether to call it good luck or bad", said one ISAS scientist of the collisions - bad luck to have been hit or good luck to have discovered something quite unexpected; no dust particles this large were expected to be thrown off by the comet.

Not unsurprisingly, there has been no time yet to analyse data in any detail. But results from the Lyman-alpha ultraviolet imaging camera confirms the variability in the hydrogen coma of Halley's comet that the Japanese team report in this issue of Nature (see p. 140). Detailed study should make it possible to measure the period of rotation of the comet. Preliminary results from the ion-spectrum analyser also indicate that a shock-like region forms on the Sun side of the comet as the solar wind is displaced around it. Ions from the comet were detected as far as $400,000 \mathrm{~km}$ away.

Suisei will continue to make observations of Halley for as long as possible. Over the next few days, Sakigake, the test probe launched in advance of Suisei, will come into position downwind of Halley to complement its observations. On board are instruments to measure plasma waves, ion temperature, velocity and density and the interplanetary magnetic field. But scientists at ISAS are already beginning to wonder what can be done next with Suisei. One of ISAS's satellites continued to function for six years and it is hoped that further useful observations can be made with the probe as it continues to circle the Sun.

Alun Anderson 